\title{
INFECÇÃO DE VIAS URINARIAS NA COM UNIDADE DE RIBEIRÃO PRETO - SP: ETIOLOGIA, SENSIBILIDADE BACTERIANAA ANTIMICROBIANOS E IMPLICAÇÕES TERAPÊUTICAS
}

\author{
URINARY TRACT INFECTION IN THE COMMUNITY OF Ribeirão PRETO, SP, BRAZIL: ETIOLOGY, \\ BACTERIAL SUSCEPTIBILITY TO ANTIMICROBIALS, AND THERAPEUTIC IMPLICATIONS
}

Corina Bueno Siqueira de Camargo ${ }^{\text {; }}$ Caroline de Cássia Pedro²; Débora Silva Lourenço²; Rosa Helena A Romaneilo Gironi ${ }^{3}$ \& Roberto Martinez ${ }^{4}$

\begin{abstract}
1Pós-graduanda do Programa de Clínica Médica. ${ }^{2}$ Aprimorandas. ${ }^{3}$ Biologista. Laboratório de Microbiologia do Hospital das Clínicas da FMRP USP, ${ }^{4}$ Docente. Departamento de Clínica Médica Faculdade de Medicina de Ribeirão Preto — USP.

Correspondência: Prof. Roberto Martinez. Faculdade de Medicina de Ribeirão Preto - USP. Av Bandeirantes, 3900. CEP 14048-900 Ribeirão Preto - SP. rmartine@fmrp.usp.br, FAX: (016) 633-669
\end{abstract}

CAMARGO CBS; PEDRO CC; LOURENÇO DS; GIRONI RHA \& MARTINEZ R. Infecção de vias urinárias na comunidade de Ribeirão Preto, SP: Etiologia, sensibilidade bacteriana a antimicrobianos e implicações terapêuticas. Medicina, Ribeirão Preto, 35: 173-178, abr./jun. 2002.

RESUMO: Este estudo teve como objetivo avaliar a etiologia da infecção do trato urinário e a susceptibilidade das bactérias mais freqüentemente isoladas da urina de pacientes atendidos em Unidades Básicas de Saúde de Ribeirão Preto, SP. A urina foi coletada entre outubro de 1996 e maio de 2000. A identificação bacteriana e o teste de sensibilidade a antimicrobianos foram realizados pelo sistema automatizado Microscan (Dade - Behring), menos nos seis meses iniciais do período acima citado, quando se recorreu à identificação bacteriológica convencional e ao método de difusão de disco.

Os agentes causais distribuíram-se em enterobactérias $(87,6 \%)$, bacilos Gram-negativos não fermentadores (1,5\%) e cocos Gram-positivos (11,9\%). De um total de 1.638 bactérias, as espécies mais prevalentes foram Escherichia coli (1108 - 67,6\%), Klebsiella pneumoniae (107$6,5 \%)$, Proteus mirabillis (67 - 4,1\%), Staphylococcus saprophyticus ( $63-3,8 \%$ ), Citrobacter koseri ( $26-1,6 \%$ ) e Enterococcus faecalis ( $25-1,5 \%$ ). A sensibilidade de $E$. coli foi baixa para ampicilina ( $45 \%$ ) e cefalotina ( $54 \%$ ), mediana para sulfametoxazol e trimetoprim (63\%) e alta para amoxicilina e clavulanato, cefuroxima, ceftriaxona, ceftazidima, gentamicina, amicacina, nitrofurantoína, norfloxacina, ácidos pipemídico e nalidíxico e ciprofloxacina (92\% - 100\%). A sensibilidade geral dos uropatógenos sugere que norfloxacina e outras quinolonas, nitrofurantoina, cefuroxima e gentamicina são opções terapéuticas adequadas para as infecções de vias urinárias na comunidade.

UNITERMOS: Infecções Urinárias. Infecções Comunitárias Adquiridas. Antibioticoterapia. Resistência Bacteriana. Bacteriúria.

\section{INTRODUÇÃO}

Infecção das vias urinárias é causa freqüente de morbidade na população, representando percentual significativo dentre as consultas em serviços médicos públicos. Com exceção das lhixas etárias extremas, predominam amplamente em mulheres, sendo incomuns em homens com idade inferior a 60 anos. São 
predisponentes dessas infecções, entre outros fatores, anomalias congênitas e litiase de vias urinárias, disfunção vesical com retenção urinária, atividade sexual nas mulheres, gravidez, prostatismo e sondagem vesical prolongada ${ }^{1,2}$. As infecções urinárias comunitárias, não complicadas têm como principais agentes etiológicos os bacilos Gram-negativos, sobressaindose Escherichia coli, Klebsiella pneumoniae e Proteus mirabilis. Enterococo e espécies de estafilococos e de estreptococos são responsáveis por fração menor de $\operatorname{casos}^{1}$. Essa distribuição tem sido mundialmente observada, contudo pode se alterar na frequiência relativa das espécies e quanto aos tipos de agentes em infecções complicadas, em pacientes com alterações persistentes de vias urinárias, infecções recidivantes ou cronificadas, sondas vesicais ou previamente tratadas ${ }^{2,3}$.

A sensibilidade das bactérias causadoras de infecções urinárias vem se reduzindo para os antimicrobianos mais usados no tratamento dos pacientes. $\mathrm{O}$ fenômeno tem sido observado em vários países, motivando avaliação continuada da susceptibilidade de microorganismos isolados da urina ${ }^{4}$. O tratamento das infecções urinárias não complicadas, principalmente em mulheres sem problemas subjacentes de importância, é, em geral, iniciado empiricamente, buscandose prescrever medicações com grande probabilidade de obtenção de cura clínica e bacteriológica. Para isso, a seleção dos antimicrobianos baseia-se prioritariamente na etiologia e na sensibilidade bacteriana relativa à comunidade dos doentes. Visando à atualização e, eventualmente, propiciar a revisão de esquemas terapêuticos, objetivou-se avaliar dados referentes a bactérias isoladas em uroculturas de pacientes de Unidades Básicas de Saúde de Ribeirão Preto - SP.

\section{MATERIAL E MÉTODOS}

No período de outubro de 1996 a maio de 2000, o Laboratório de Microbiologia do Hospital das Clínicas da Faculdade de Medicina de Ribeirão Preto-USP recebeu para cultura 7.641 amostras de urina de pacientes com diagnóstico clínico de infecção urinária, após atendimento em Unidades Básicas de Saúde de Ribeirão Preto SP. Processada em período máximo de quatro horas depois da coleta, a urina foi semeada com alça calibrada em agar CLED e incubada a $37^{\circ} \mathrm{C}$ durante $20 \mathrm{~h}$. Nas placas com crescimento bacteriano, foi estimado o número de colônias por $\mathrm{mL}$ de urina. A identificação das bactérias isoladas e o teste de sensibilidade a antimicrobianos foram realizados em painéis do sistema de automação Microscan (Dade Behring). Nos seis meses iniciais do estudo, entretanto, a identificação foi feita por meio de testes bacteriológicos convencionais e o antibiograma por difusão de disco em placas com meio Mueller-Hinton, de acordo com normas estabelecidas pelo NCCLS dos Estados Unidos 5 .

Os resultados das amostras de urina com crescimento bacteriano igual ou superior a 10.000 colônias $/ \mathrm{mL}$ foram retrospectivamente compilados. Quando existiam duas ou mais uroculturas positivas, do mesmo paciente, incluiu-se no estudo apenas a primeira. Analisou-se o teste de sensibilidade somente das espécies mais isoladas e, com relação a E. coli, apenas os antibiogramas dos meses ímpares. Diferenças na composição de antimicrobianos entre painéis usados também foram causas para que o número de amostras bacterianas testadas para cada droga fosse inferior ao total de isolamentos de cada espécie. Ácido nalidíxico e ácido pipemídico foram testados somente pelo método de difusão de disco.

\section{RESULTADOS}

Em 1.638 amostras de urina ocorreu isolamento de bactérias em número superior a $10.000 / \mathrm{mL}$, cuja distribuição é apresentada na Tabela I. Predominaram as enterobactérias, destacando-se $E$. coli, que causou dois terços das infecções, espécies de Klebsiella, principalmente $K$. pneumoniae, e $P$. mirabilis. Espécies de Citrobacter, Enterobacter, Serratia, Morganella, Providentia e Proteus não mirabilis foram encontradas com menor freqüência. Pseudomonas aeruginosa e outros bacilos Gram-negativos não fermentadores representaram apenas $1,5 \%$ dos isolamentos. Aproximadamente $12 \%$ das bactérias foram identificadas como cocos Gram-positivos, em particular Staphylococcus saprophïticus e Enterococcus faecalis, embora, também, diversas espécies de estreptococos a - maioria dos grupos B, D e viridans e outros estafilococos e enterococos tenham sido identificadas.

A Tabela II mostra a sensibilidade a antimicrobianos das bactérias Gram-negativas e Gram-positivas mais isoladas, correspondendo a aproximadamente $85 \%$ do total. Salienta-se a significativa resistência de E.coli para cefalotina, ampicilina e sulfametoxazol trimetoprim (cotrimoxazol); de K. pneumoniae à ampicilina e a vários quimioterápicos de vias urinárias; de $P$. mirabilis à ampicilina e nitrofurantoína; de $S$. saprophyticus para ampicilina a ácido nalidíxico. $E$. faecalis, considerado naturalmente resistente a 
Tabela I - Bactérias isoladas da urina de pacientes de Unidades Básicas de Saúde - Ribeirão Preto, SP- 10/96 a 05/00

\begin{tabular}{|l|r|r|}
\hline BACTÉRIA & $\boldsymbol{N}^{\circ}$ & $\mathbf{\%}$ \\
\hline Enterobactérias & 1418 & $87,6 \%$ \\
\hline Escherichia coli & 1108 & 67,6 \\
\hline Klebsiella pneumoniae & 107 & 6,5 \\
\hline Klebsiella spp (não pneumoniae) & 15 & 0,9 \\
\hline Proteus mirabilis & 67 & 4,1 \\
\hline Citrobacter koseri & 26 & 1,6 \\
\hline Citrobacter spp. (não-koseri) & 22 & 1,3 \\
\hline Enterobacter cloacae & 22 & 1,3 \\
\hline Enterobacter aerogenes & 21 & 1,3 \\
\hline Outras Enterobactérias & 30 & 1,8 \\
\hline Bacilos Gram-negativos não fermentadores & 25 & $1,5 \%$ \\
\hline Pseudomonas aeruginosa & 9 & 0,5 \\
\hline Acinetobacter spp. & 11 & 0,7 \\
\hline Outros não fermentadores & 5 & 0,3 \\
\hline Gram-positivos & 195 & $11,9 \%$ \\
\hline Staphylococcus saprophyticus & 63 & 3,8 \\
\hline Enterococcus faecalis & 25 & 1,5 \\
\hline Enterococcus spp. (não-faecalis) & 3 & 0,2 \\
\hline Streptococcus agalactiae & 22 & 1,3 \\
\hline Outros estreptococos & 38 & 2,3 \\
\hline Staphylococcus aureus & 16 & 1,0 \\
\hline Outros Estafilococos coagulase negativos & 28 & 1,7 \\
\hline TOTAL & $\mathbf{1 6 3 8}$ & $\mathbf{1 0 0 , 0}$ \\
\hline
\end{tabular}

cefaloporinas, mostrou-se totalmente sensível à ampicilina e à nitrofurantoína. No conjunto das bactérias constantes da Tabela II, verificou-se atividade elevada (acima de 90\% de susceptíveis) para amoxicilina e clavulanato, cefuroxima, ceftriaxona, ceftazidima, gentamicina, amicacina, ciprofloxacina e norfloxacina. Apresentaram atividade moderada $(60 \%$ a $85 \%$ de amostras sensíveis) cefalotina, cotrimoxazol, ácidos pipemídico e nalidíxico e nitrofurantoína. Ampicilina teve a pior atuação in vitro (39\%).

\section{DISCUSSÃO}

No período de estudo, as uroculturas requisitadas para pacientes do SUS do Município de Ribeirão Preto foram centralizadas em laboratório de hos- pital universitário. Em grande parte, as identificações bacterianas e os testes de sensibilidade a antimicrobianos foram realizados em sistema de automação em Microbiologia que, por ser amplamente utilizado nos Estados Unidos da América e em países europeus, facilita o cotejamento dos resultados. Essas condições proporcionaram a presente análise sobre agentes bacterianos de infecções urinárias de pacientes ambulatoriais da comunidade de Ribeirão Preto - SP.

A distribuição relativa das bactérias isoladas favoreceu as enterobactérias, principalmente E. coli, Klebsiella e Proteus mirabilis, as quais somadas a S.saprophyticus e E. faecalis representaram cerca de $85 \%$ do total. Tal distribuição é semelhante ao verificado em estudos sobre infecções urinárias comunitárias, no Brasil e em outros países ${ }^{7,8}$. Assemelha-se, também, ao constatado cerca de vinte anos antes na mesma comunidade ${ }^{9}$.

Em 103.223 casos norte-americanos de infecção não complicada, $E$. coli foi o principal agente em $72 \%$ das mulheres com 15 a 50 anos e em 53\% daquelas com mais de 50 anos, seguindo-se Klebsiella spp., Proteus spp., Enterobacter spp., e outros bacilos Gram-negativos. Nesse mesmo estudo ${ }^{7}$, enterococo e $S$. saprophyticus também foram encontrados como agentes causais de infecções de vias urinárias, sendo suas freqüências mais elevadas, respectivamente, em mulheres mais idosas ou mais jovens. Por outro lado, estafilococos coagulase negativos e outras bactérias que colonizam a pele e a mucosa vaginal podem ser apenas contaminantes da urocultura ${ }^{10}$. O predomínio universal de E. coli e de outras enterobactérias, como causa de infecções urinárias não complicadas, tem sido atribuído à colonização da uretra distal por microorganismos de origem intestinal. Nas infecções urinárias do sexo masculino e em todas as outras consideradas complicadas, as enterobactérias também representam a etiologia principal, porém com maior participação de Klebsiella, Proteus, Enterobacter e Serratia, além de Pseudmonas aeruginosa e outros bacilos Gramnegativos não-fermentadores ${ }^{11}$. 
Tabela II - Sensibilidade in vitro a antimicrobianos das bactérias mais freqüientemente isoladas da urina de pacientes do município de Ribeirão Preto - SP (outubro/96 a maio/2000).

\begin{tabular}{|c|c|c|c|c|c|c|c|c|c|c|c|c|c|c|}
\hline Bactérias & AMP & $\mathrm{AMC}$ & $\mathrm{CF}$ & CXM & $\mathrm{CRO}$ & CAZ & GEN & AMI & CIP & NOR & PP & NA & NIT & SFT \\
\hline $\begin{array}{l}\text { E.coli } \\
\mathrm{N}^{\circ} \text { testado }\end{array}$ & 530 & 205 & 421 & 404 & 250 & 215 & 496 & 440 & 350 & 501 & 138 & 176 & 504 & 533 \\
\hline Sensibilidade (\%) & 45 & 92 & 54 & 95 & 100 & 99 & 98 & 99 & 96 & 97 & 96 & 96 & 96 & 63 \\
\hline $\begin{array}{l}\text { K.pneumoniae } \\
\mathrm{N}^{\circ} \text { testado }\end{array}$ & 107 & 44 & 104 & 77 & 54 & 57 & 103 & 101 & 84 & 104 & 28 & 34 & 102 & 107 \\
\hline Sensibilidade (\%) & 1 & 89 & 73 & 89 & 98 & 98 & 92 & 98 & 93 & 85 & 64 & 68 & 65 & 72 \\
\hline $\begin{array}{l}\text { P. mirabilis } \\
\mathrm{N}^{\circ} \text { testado }\end{array}$ & 65 & - & 63 & 47 & 31 & 15 & 53 & 44 & 32 & 61 & 15 & 22 & 62 & 64 \\
\hline Sensibilidade (\%) & 35 & - & 89 & 96 & 100 & 100 & 92 & 98 & 100 & 98 & 100 & 86 & 2 & 67 \\
\hline $\begin{array}{l}\text { s. saprophyticus } \\
\mathrm{N}^{\circ} \text { testado }\end{array}$ & 60 & 18 & 53 & 25 & 10 & - & 59 & 35 & 41 & 60 & 17 & 17 & 60 & 59 \\
\hline Sensibilidade (\%) & 30 & 83 & 91 & 100 & 100 & - & 100 & 100 & 95 & 93 & 100 & 6 & 98 & 93 \\
\hline $\begin{array}{l}\text { E. faecalis } \\
\mathrm{N}^{\circ} \text { testado }\end{array}$ & 24 & - & - & - & - & - & - & - & 22 & 24 & - & - & 25 & \\
\hline Sensibilidade (\%) & 100 & - & - & - & - & - & - & - & 77 & 79 & - & - & 100 & \\
\hline otal testado & 786 & 267 & 64 & 553 & 345 & 287 & 711 & 620 & 529 & 750 & 198 & 249 & 753 & 763 \\
\hline Sensíveis (nº) & 307 & 242 & 409 & 521 & 343 & 283 & 688 & 613 & 501 & 707 & 165 & 212 & 637 & 509 \\
\hline$\%$ & 39,1 & 90,6 & 63,8 & 94,2 & 99,4 & 98,6 & 96,8 & 98,9 & 94,7 & 94,3 & 83,3 & 85,1 & 84,6 & 66,7 \\
\hline
\end{tabular}

$\mathrm{AMP}=$ ampicilina; $\mathrm{AMC}=$ amoxicilina ácido clavulânico; $\mathrm{CF}=$ cefalotina; $\mathrm{CXM}=$ cefuroxima; $\mathrm{CRO}$ ceftriaxona; $\mathrm{CAZ}=$ ceftazidima; GEN= gentamicina, AMI amicacina; $\mathrm{CIP}=$ ciprofloxacina; NOR norfloxacina; $\mathrm{PP}$ ácido pipemidico; NA= ácido na NIT= nitrofurantoina;

$\mathrm{SFT}=$ sulfametoxazol-trimetropim (cotrimoxazol)

Alertas sobre o aumento da resistência de patógenos urinários a antimicrobianos já datam de algumas décadas ${ }^{9,12}$. São as medicações mais amplamente utilizadas no combate a diversos tipos de infecções, como ampicilina, cefalosporinas de primeira geração e cotrimoxazol, que sofreram a maior redução de eficácia, correspondendo, neste estudo, a, respectivamente, $55 \%, 46 \%$ e $37 \%$ de amostras resistentes de $E$. coli. Nos últimos anos, nos Estados Unidos, a resistência de E. coli, in vitro, atingiu 30\% - 40\% para ampicilina e $20 \%-30 \%$ para cefalotina ${ }^{13}$. Em estudo brasileiro $^{14}, 54 \%$ das amostras de E. coli oriundas de doentes ambulatoriais mostraram-se resistentes à ampicilina. Em período recente, a resistência de $E$. coli ao cotrimoxazol situava-se entre $12 \%$ a $19 \%$, na Inglaterra, França, Bélgica, Holanda e Canadá e entre 31 a $60 \%$, na Espanha, Israel e Bangladesh ${ }^{15,16}$. Índices ainda mais elevados de resistência de enterobactérias à ampicilina e ao cotrimoxazol são observados em países subdesenvolvidos. Resistência de E. coli a três ou mais drogas, especialmente ampicilina, cetalotina e cotrimoxazol, chegou a $7^{\circ} \mathrm{o}$ das amostras de norte-americanos, porém foi mais encontrada em mulheres com mais de 65 anos, homens e pacientes hospitaIizados ${ }^{4}$. Drogas relacionadas ao grupo das quinolonas, tanto as precursoras, como as recentes, permanecem atuantes contra a maioria das amostras de enterobactérias de origem comunitária, sendo as taxas de resistência iii vitro de $E$. coli para norfloxacina e ciprofloxacina inferiores a $20 \%$, tanto em diversas regiões geográficas do mundo ${ }^{14,15,17}$, como no presente estudo. É também baixo, não superior a 11\%, o percentual de amostras de E. coli resistentes à nitrofurantoína, embora esse quimioterápico esteja há longo tempo no arsenal terapêutico ${ }^{18}$. De modo geral, outras enterobactérias foram mais resistentes a antimicrobianos do que E. coli. Pseudomonas aeruginosa e outros bacilos Gram-negativos não fermentadores apresentam resistência intrínseca maior do que as enterobactérias, porém são incomuns como agentes de processos infecciosos em pessoas não hospitalizadas.

O tratamento das infecções de vias urinárias visa não somente obter cura clínica, como eliminar os agentes causais, prevenindo recorrências. Os esque- 
mas terapêuticos levam em consideração a eficácia antimicrobiana e a excreção urinária das drogas disponíveis, além de toxicidade, custo e comodidade posológica. Ainda se prioriza a associação sulfametoxazol e trimetoprim, ou somente esta última droga, para o tratamento empírico de infecções urinárias, baixas, em pacientes da comunidade, particularmente nas mulheres. Existem evidências de que com o uso dessas medicações, durante três dias, consegue-se cura clínica e bacteriológica na maioria dos casos de cistite ${ }^{19}$. Contudo, a recomendação é válida para casos não complicados, na ausência de gravidez, e em áreas onde a prevalência de resistência de $E$. coli ao cotrimoxazol não supere $20 \%{ }^{19}$. No Brasil, a despeito da maior resistência a esse medicamento, altas taxas de sucesso clínico e bacteriológico têm sido alcançadas com tratamentos de sete dias de duração em infecções não complicadas, em mulheres ${ }^{20,21}$. Ampicilina ou amoxicilina representam alternativas com menor eficiência para o tratamento empírico, pois os patógenos urinários são menos sensíveis, há menor cura bacteriológica, e o percentual de recorrências é maior 11,22,23. Quinolonas constituem boa opção terapêutica para infecções urinárias não complicadas, por serem igualmente ou mais eficazes do que cotrimoxazol na obtenção de cura clínica e prevenção de recorrências, conseguindo-se elevados índices de sucesso no Brasil com norfloxacina e outras drogas do mesmo grupo químico ${ }^{20,25}$. No tratamento das pielonefrites podem ser empregadas norfloxacina ou antibióticos, por via oral, durante 10 a 14 dias, recorrendo-se, em casos graves ou complicados, à terapia endovenosa com cefalospo- rinas de primeira à terceira geração, cotrimoxazol, fluorquinolonas, gentamicina ou ampicilina ${ }^{10,19}$.

A boa atuação contra a maioria das bactérias isoladas sugere que norfloxacina, ácido pipemídico e outras drogas quinolônicas sejam, atualmente, a melhor opção para o tratamento empírico das infecções urinárias comunitárias, em Ribeirão Preto. Cefurosima e ácido clavulânico de amoxicilina também representam boas opções para terapia oral ou parenteral, porém têm custo mais elevado. Tratamento parenteral domiciliar pode ser efetuado com gentamicina por via intramuscular. Cotrimoxazol, nitrofurantoína e cefalosporinas de primeira geração, menos eficazes in vitro, podem ainda constituir alternativas válidas para infecções não complicadas. Apoiando essa conduta, constatou-se que bactérias urinárias de casos simples da comunidade são discretamente mais sensíveis do que aquelas isoladas de amostras enviadas à laboratórios de Microbiologia ${ }^{18}$. Para pacientes com pielonefrite mais grave ou complicadas infecções de vias urinárias, o perfil de susceptibilidade bacteriana, apresentado na Tabela II sugere que o tratamento possa ser iniciado por via endovenosa com cefuroxima, ceftriasona, gentamicina ou ciprofloxacina. Em tais casos, é indispensável a realização da urocultura e do antibiograma, em vista da necessidade de uma terapia rapidamente efetiva, proporcionada pela escolha dirigida do antimicrobiano. A infecção enterocócica, por exemplo, deve ser combatida com ampicilina ou com associação de ampicilina e gentamicina . Também é necessário observar as condições especiais do paciente, incluindo crianças, idosos e gestantes, que requerem abordagem diferenciada.

CAMARGO CBS; PEDRO CC; LOURENÇO DS; GIRONI RHA \& MARTINEZ R. Urinary tract infection in the community of Ribeirão Preto, SP, Brazil: Etiology, bacterial susceptibility to antimicrobials, and therapeutic implications. Medicina, Ribeirão Preto, 35: 173-178, apr./june 2002.

ABSTRACT: This study intended to evaluate the etiology of urinary tract infection and the antimicrobial susceptibility of the bacteria more frequently isolated of the urine of patients assisted in Primary Health Care Units of Ribeirão Preto, SP, Brazil. The urine was collected between October 1996 and May 2000. The identification and antimicrobial susceptibility of the isolated bacteria were made in the automated system Microscan ( Dade - Behring ), except in the first six months of the study, when conventional bacteriological identification and disk diffusion methods were used causative agents were distributed in Enterobacteriaceae $(87,6 \%)$, nonfermentative Gram negative bacilli $(1,5 \%)$, and Gram - positive cocci $(11,9 \%)$. Most prevalent species in a total of 1638 bacteria were Escherichia coli $(1108-67,6 \%)$, Klebsiella pneumoniae (107 - 6,5\%), Proteus mirabilis ( $67-4,1 \%$ ), Staphylococcus saprophyticus ( $63-3,8 \%$ ), Citrobacter koseri $(26-1,6 \%)$, and Enterococcus faecalis $(25-1,5 \%)$. The susceptibility of E. coli was low for ampicillin (45\%) and cephalothin ( $54 \%$ ), median for trimethoprim - sulphamethoxazole ( $63 \%$ ), and high for amoxicillin - clavulanate, cefuroxime, ceftazidime, gentamicin, amikacin, nitrofurantoin, norfloxacin, pipemidic acid, nalidixic acid and ciprofloxacin (92\% - 100\%). The overall sensitivities of uropathogens suggest that norfloxacin and other quinolones, nitrofurantoin, cefuroxime, and gentamicin are adequate therapeutic options for community urinary tract infections.

UNITERMS: Urinary Tract Infections. Community Acquired Infections. Antimicrobial Therapy. Bacterial Resistance. Bacteriuria. 


\section{RFFERÊNCIAS BIBLIOGRÁFICAS}

1 - BARNETT BJ \& STEPHEN DS. Urinary tract infection : an overview. Am J Med Sci 314: 245 —249, 1997.

2- STAMM WE; HOOTON TM; JOHNSON JR; JOHNSON C; STAPLETON A; ROBERTS PL; MOSELLY SL \& FIHN SD. Urinary tract infections: from pathogenesis to treatment. J Infect Dis 159 400-406, 1989.

3 - KUNIN CM. Nosocomial urinary tract infections and the indwelling catheter: what is new and what is true?. Chest 120 : 10-12, 2001.

4- SAHM DF; THORNSBERRY C; MAYFIELD DC; JONES ME \& KARLOWSKY JA. Multidrug - resistant urinary tract iso lates of Escherichia coli: prevalence and patient demograph ics in the United States in 2000. Antimicrob Agents Chemother 45: 1402-1406, 2001.

5 - NATIONAL COMMITTEE FOR CLINICAL LABORATORY STANDARDSApí0ved Standards M - A Standard Meth ods for dilution antimicrobial susceptibility tests for bacteria that grow aerobically. 3 ed. Vilianova, PA, NCCLS, 1993.

6 - LIMA CMH; QUEIROZ Dc; SILVA CMC; MOREIRA FC; NOSHIKAWALY; SILVAMP; SHIOZAWAPC; BIANCHI MRE; PERUGINI M; PELISSON VLC; ABBONDANZA RMQ \& CARRARA FE. Incidência, etiologia de infecções urinárias e perfil de sensibilidade das enterobactêrias isoladas da usi na no Ambulatório do Hospital das Clínicas (AHC) de Lon drina-PR. In: Anais, XX Congresso Brasileiro de Microbiologia, Salvador-BA, p58, 1999.

7- GUPTAK; SAHM DF; MAYFIELD D \& STAMM WE. Antimicro bial resistance among uropathogens that cause community - acquired urinary tract infection in women: a nationwide analysis. Clin Infect Dis 33: 89-94, 2001.

8 - KOMAROFF AL. Acute dysuria in women. N Engl J Med 310: 368-375, 1984.

9 - POLETTO MR; MARTINEZ R \& LEVY CE. Infecção das vias urinárias em 2154 pacientes: germes isolados e sensibilida de a antimicrobianos. In: Anais, XIX Congresso da Soci edade Brasileira de Medicina Tropical, Rio de Janeiro - RJ, pl37I38, 1983.

IO-RUBIN RH; BEAM JR. TR \& STAMM WE. An approach to evaluating agents in the treat ment of urinary tract infection. Clin Infect Dis 14: S246—S251, 1992. Suppl 2.

11 - BACHELLER CD \& BERNSTEIN JM. Urinary tract infections. Med Clin North Am 81:719-730, 1997

12 - GRÜNEBERG RN. Antibiotic sensitivitíes of urinary patho gens. J Clin Pathol 33 853-856, 1980.

13- STAMM WE \& NORRBY SR. Urinary tract infections disease panorama and challenges. J Infect Dis 183: S1-S4, 2001. Suppl. 1.
14 - BERDICHEYSKI RH; ZANARDO JC; SOIBELMAN M \& ANTONELLO 1 Prevalência de uropatógenos e sua resis tência aos antimicrobianos em uroculturas realizadas no Hospital São Lucas da PUCRS. Rev Assoc Med RGS 42: 137-142, 1998.

15- GUPTAK, HOOTON TM \& STAMM WE. Increasing antimicro bial resistance and the management of uncomplicated com munity-acquired urinary tract infections. Ann Intern Med 135: 41-50, 2001.

16- VU-THIEN H Sensibilité aux antibiotiques des bactéries isolées dans les infections urinaires em pédiatrie. Arch Pédiatr 5: 266-268, 1998. Suppl.3

17- AHMED AA; OSMAN H; MANSOUR AM; MUSA HA; AHMED $A B$; KARRAR Z \& HASSAN HS. Antimicrobial agent resis tance in bacterial isolates from patients with diarrhea and urinary tract infection in the Sudan. Am J Trop Med Hyg 63: 259-263, 2000.

18- BAERHEIN A. Empirical treatment of uncomplicated cystitis. Keep it simple. Br Med J 323: 1197-1198, 2001.

19-WARREN JW; ABRUTYN E; HEBEL JR; JOHNSON JR; SCHAEFFER AJ \& STAMM WE. Guidelines for antimicrobial treatment of uncomplicated acute bacterial cystitis and acute pyelonephritis in women. Clin Infect Dis 29: 745-758, 1999.

20-ALDRIGHI J; SILVAAA; SAAB NETO JA; PAGANI JP; REMIJIO NETO $J$ \& COIMBRA MG. Estudo multicêntrico da eficácia e segurança da lomefloxacina no tratamento da cistite em mulheres. Rev Bras Med 53: 290-294, 1996.

21 - CLAROJA; REIS CU; BANDEIRAS; LIMAM; SCAFIC \& NETTO JUNIOR NR. Tratamento dose única com $800 \mathrm{mg}$ de pefloxacina versus tratamento de sete dias com norfioxacina e co-trimoxazol na infecção urinária baixa. J Bras Ginecol 104: 51-53, 1994.

22 - PIMENTAWP; PEIXOTO E; AOKI R; MICHELIN LA; PADOVANI CR \& MONTELI AC. Bacteriúria em pacientes com diabetes mellitus. J Bras Patol 33: 189-195, 1997.

23- RIBEIRO RM; GUIDI HGC; BRACHER ESB \& MOTTAEV. Trata mento em dose única de infecção do trato urinário baixo não complicada estudo randomizado com fosfomicina trometamol e amoxicilina. J Bras Ginecol 102: 47-49, 1992.

24 - SPERIDIÃO 5. Avaliação multicêntrica do norfloxacino no tratamento das infecções do trato urinário não complica das. Rev Bras Med 53: 301-303, 1996.

25 - FUCS M. Avaliação do uso de ofloxacina em dose única diária por três dias no tratamento das infecções urinárias não complicadas. Arq Bras Med 68: 289-292, 1994.

Recebido para publicaçã em 22/04/2002

Aprovado para publicação em 28/06/2002 